\title{
Levantamento etnobotânica e utilização de plantas medicinais na comunidade Moura, Bananeiras-PB
}

\author{
Ethnobotany survey and use of medicinal plants in the community of Moura, Bananeiras-PB
}

\author{
Alian Cássio Pereira Cavalcante', Adailza Guilherme da Silva² \\ 1,2Universidade Federal da Paraíba - PB - Brasil
}

\begin{abstract}
Resumo
O reconhecimento e a valorização do saber local sobre as plantas medicinais são fundamentais em comunidades rurais, pois os remédios caseiros surgem como alternativa de cura. Desta forma objetivou-se realizar um levantamento etnobotânicos das plantas medicinais cultivadas e utilizadas pelos moradores da comunidade Moura zona rural de Bananeiras-PB. O estudo foi realizado no mês de novembro de 2013, os dados foram obtidos através e questionários semiestruturado completadas por entrevistas livres e conversas informais com as pessoas da comunidade. O número expressivo de espécies de plantas medicinais citadas evidencia o rico conhecimento etnobotânico da população local. As espécies identificas na comunidade suprem uma série de necessidades básicas dos moradores sem deixar necessitar de serem utilizados remédios farmacêuticos.
\end{abstract}

Palavras-chave: Etnobotânica, conhecimento popular, fitoterápicos.

\begin{abstract}
The recognition and appreciation of local knowledge about medicinal plants are essential in rural communities, because home remedies are an alternative healing. Thus we aimed to conduct an ethnobotanical survey of medicinal plants cultivated and used by community residents Moura rural Bananeiras-PB. The study was conducted in November 2013, data were obtained through semi-structured questionnaires and supplemented by free interviews and informal conversations with people in the community. The highest number of species of medicinal plants cited evidences the rich ethnobotanical knowledge of the local population. You identify the species in the community supplying a number of basic needs of the residents while requiring use pharmaceutical drugs.
\end{abstract}

Keywords: Ethnobotany, popular knowledge, herbal. 


\section{INTRODUÇÃO}

As plantas medicinais, tiveram a sua origem desde o ano 3000 a.C. pelos povos Chineses que dedicava-se ao seu cultivo (OLIVEIRA; ARAUJO, 2007). O uso de plantas medicinais na recuperação da saúde tem evoluído ao longo dos tempos desde as formas mais simples de tratamento local, provavelmente utilizada pelo homem das cavernas, até as formas tecnologicamente sofisticadas da fabricação industrial utilizada pelo homem moderno (LORENZ; MATOS, 2002).

Caravaca (2000) explica que cada povo possui sua própria lista de ervas medicinal, plantas comuns típicas da região em que vivem e das quais o emprego é transmitido de geração a geração. $\mathrm{O}$ reconhecimento e a valorização do saber local sobre as plantas medicinais são fundamentais em comunidades rurais, pois os remédios caseiros surgem como alternativa de cura, muitas vezes a única devido à falta de outros recursos para cuidar da saúde (CUNHA; BORTOLOTTO, 2011).

Ao resgatar o conhecimento e as técnicas terapêuticas é uma maneira de deixar registrado um modo de aprendizado informal que contribui para a valorização da medicina popular, além de gerar informações sobre a saúde da comunidade local (PILLA; AMOROZO; FURLAN, 2006).

Os estudos etnobotânicos são importantes, pois permitem avaliar de que forma os moradores reúnem conhecimentos trazidas de seus locais de origem e transmitidas às novas gerações. Desta forma objetivou-se realizar um levantamento etnobotânicos das plantas medicinais cultivadas e utilizadas pelos moradores da comunidade Moura localizada zona rural de bananeiras-PB.
O estudo foi realizado na comunidade Moura, zona rural do município de Bananeiras -PB, (Figura 1), no mês de novembro de 2013. A região é localizada no Brejo paraibano, apresenta baixa densidade populacional, constituído de 41 moradores, reunidos em 13 famílias, sendo a organização social centralizada nas famílias e no grau de parentesco.

Os dados foram obtidos através de questionários semiestruturado completadas por entrevistas livres e conversas informais com as pessoas da comunidade, semelhante a pesquisa realizada por (SANTOS et al, 2012). Antes das entrevistas foi explicado o objetivo da pesquisa e solicitado à permissão pelos entrevistados para registrar as informações fornecidas.

O questionário foi constituído de questões sobre faixa etária de idade, gênero, aceitação das plantas medicinais pelas famílias, levantamento das plantas cultivadas e utilizadas, plantas mais citadas, espécies e famílias mais citadas, parte mais utilizada, origem do conhecimento e a finalidade de cultivar as plantas medicinais.

Os dados foram tabulados de forma qualiquantitativa, utilizando-se o Microsoft Office / Word/ Excel 2013 para a construção de tabelas e gráficos, e câmara digital modelo (Canon Power Shot SX 160 IS) para registro das imagens.

\section{RESULTADOS E DISCUSSÕES}

A Faixa etária de idade dos informantes variou de 28 a 60 anos, com $50 \%$ dos entrevistados acima de 40 anos. A importância de conhecer a faixa etária no domínio desse tipo de conhecimento

\section{METODOLOGIA}

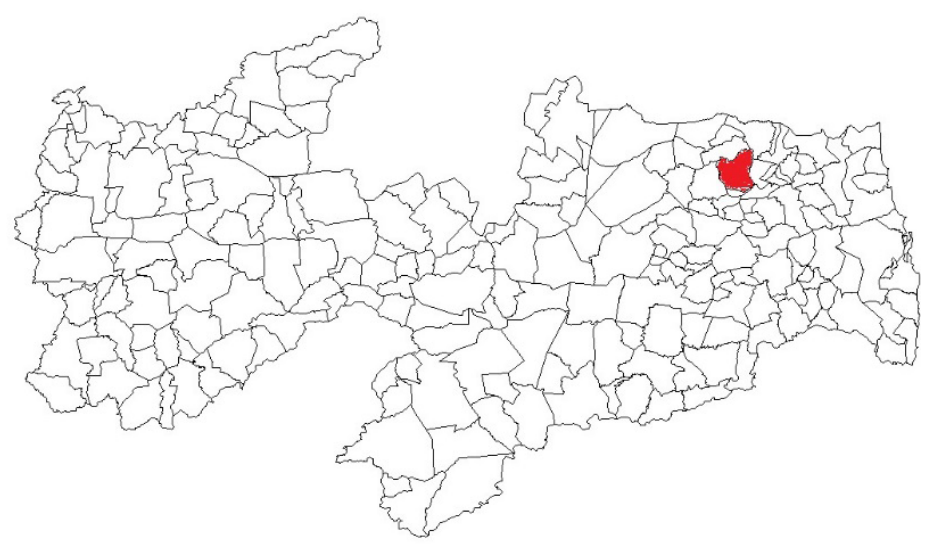

Fonte: http://geografiasociedadeemeioambiente.blogspot.com.br/

Figura 1. Localização do município de Bananeiras no estado da Paraíba. 
é muito importante. Segundo Phillips;Gentry (1993), pessoas idosas conhecem um maior numero de plantas medicinais. Em relação ao gênero dos entrevistados $56 \%$ pertencem ao sexo feminino e $44 \%$ masculino.

Quando questionados sobre a aceitação das plantas medicinais pela família (Figura 2), 85\% dos entrevistados afirmam terem muita aceitação, na qual a maioria dos familiares usam as plantas medicinais e possuem até preferência pelas mesmas, pois são acessíveis economicamente e naturais já $15 \%$ afirma ser regular, por não utilizarem com tanta frequência.
As partes das plantas mais utilizadas pelos moradores como medicina caseira foram às folhas, geralmente para a preparação de chás, por decocção seguida dos frutos e raízes. Em diversos trabalhos sobre plantas medicinais as folhas também é a parte mais utilizadas como por (JESUS et al, 2009), (PILLA, AMOROZO, FURLAN, 2006) e (CUNHA, BORTOLOTTO, 2011).

Quando indagados com quem adquiriram o conhecimento de cultivar e utilizas as plantas para fins medicinais (Figura 4), 77\% afirmaram ter sido com os pais, $15 \%$ com os avós, sendo um conhecimento que vem sendo passado de gera-

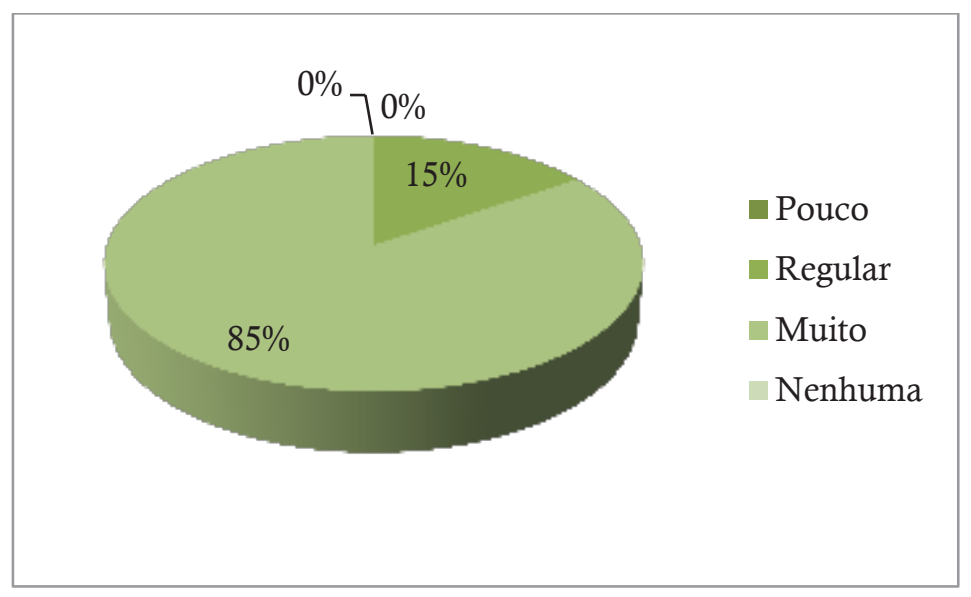

Figura 2. Aceitação das plantas medicinais pelas famílias da comunidade Moura, Bananeiras - PB, 2013.

O número expressivo de espécies de plantas medicinais citadas evidencia o rico conhecimento etnobotânico da população local. Sendo citadas 45 espécies pertencentes a 31 famílias de plantas medicinais existente na comunidade (Tabela 1 ).

Entre as famílias mais citadas destaca-se a Laminaceae, Anacardiaceae, Rutaceae, Verbenaceae, Rubiaceae, Myrtaceae e Euphorbeaceae. A mais citada pelos moradores da comunidade foi Lamiaceae, que apresenta porte herbáceo semelhante a trabalho realizado por (PILLA, AMOROZO, FURLAN, 2006) identificada no distrito de Martim Francisco, município de Mogi-Mirim, SP, Brasil.

As mais citadas foram arruda (Ruta graveolens L.), erva-cidreira (Lippia alba (Mill) N.E. Br.), louro (Ocimum sp.), romã (Punica granatum), capim santo (Cymbopogon citratus (DC) Stapf.), hortelã miúda (Mentha piperita L.), mentruz (Chenopodium ambrosioides L.) e laranjeira (Citrus $X$ sinensis (L.) Osbeck) (Figura 3). ção para geração e $8 \%$ dos vizinhos, onde existe também a troca de mudas e saberes. (BRITO, SENNA-VALLE, 2011) também identifica que a maioria do conhecimento sobre as plantas medicinais é transmitida ao longo dos anos por pais, avós e vizinhos, demonstrando uma rica herança cultural sobre plantas medicinais nessa localidade.

Quando indagados sobre a finalidade de cultivas as ervas medicinais, $100 \%$ dos participantes afirmam ser para uso terapêutico no consumo interno da família, portanto não comercializam para fins econômicos. De acordo com Cavalcante et al (2011), as plantas medicinais além de serem cultivadas para o consumo familiar também pode ser comercializada com fins lucrativos e servir como fonte complementar da renda familiar.

\section{CONCLUSÕES}

As espécies identificas na comunidade suprem uma série de necessidades básicas dos 
Tabela 1. Plantas medicinais utilizadas pelos moradores da comunidade Moura, Bananeiras-PB.

\begin{tabular}{|c|c|c|c|c|c|}
\hline Família & $\begin{array}{l}\text { Nome } \\
\text { popular }\end{array}$ & Nome científico & $\begin{array}{c}\text { Parte } \\
\text { utilizada }\end{array}$ & Indicação & $\begin{array}{l}\text { Número } \\
\text { de } \\
\text { citações }\end{array}$ \\
\hline Lauraceae & Abacate & Persea americana Mill. & Folhas & $\begin{array}{l}\text { Diurético, } \\
\text { laxante e } \\
\text { digestivo }\end{array}$ & 2 \\
\hline Malpighiaceae & Acerola & Malpighia glabra & Fruto & Infecções & 3 \\
\hline Lamiaceae & Alecrim & Rosmarinus officinalis $L$. & Folhas & $\begin{array}{l}\text { Indigestão, tosse, } \\
\text { calmante, } \\
\text { tontura }\end{array}$ & 3 \\
\hline Fabaceae & Angico & $\begin{array}{c}\text { Anadenanthera colubrina } \\
\text { (Vell.) }\end{array}$ & $\begin{array}{l}\text { Casca do } \\
\text { caule }\end{array}$ & $\begin{array}{l}\text { Resfriado, } \\
\text { catarro, tosse, } \\
\text { inflamação }\end{array}$ & 7 \\
\hline Anacardiaceae & Aroeira & $\begin{array}{c}\text { Myracrodruon urundeuva } \\
\text { (Engl.) }\end{array}$ & $\begin{array}{l}\text { Casca do } \\
\text { caule }\end{array}$ & $\begin{array}{l}\text { Gastrite, } \\
\text { cicatrizante, } \\
\text { tosse }\end{array}$ & 2 \\
\hline Rutaceae & Arruda & Ruta graveolens $L$. & Folhas & Cólica e derrame & 9 \\
\hline Liliaceae & Babosa & Aloe vera (L.) Burm.f. & Folhas & $\begin{array}{l}\text { Câncer e } \\
\text { cicatrizante }\end{array}$ & 8 \\
\hline Apocynaceae & $\begin{array}{l}\text { Boa noite } \\
\text { branca }\end{array}$ & $\begin{array}{l}\text { Catharanthus } \\
\text { roseus var.albus G.Don }\end{array}$ & Flores & Tosse & 2 \\
\hline Anacardiaceae & Caju roxo & $\begin{array}{c}\text { Anacardium occidentale } \\
\text { L. }\end{array}$ & Casca & $\begin{array}{l}\text { Cicatrizante, } \\
\text { inflamação, } \\
\text { tosse, diabetes }\end{array}$ & 7 \\
\hline Poaceae & $\begin{array}{l}\text { Capim } \\
\text { santo }\end{array}$ & $\begin{array}{l}\text { Cymbopogon citratus } \\
\text { (DC) Stapf. }\end{array}$ & Folhas & $\begin{array}{l}\text { Dor de barriga, } \\
\text { diarreia, e febre }\end{array}$ & 9 \\
\hline Oxalidaceae & Carambola & Averrhoa carambola & Frutos & $\begin{array}{c}\text { Febre, } \\
\text { estimulador de } \\
\text { apetite }\end{array}$ & 1 \\
\hline Cactaceae & Cardeiro & Cereus jamacaru DC & Caule & Hemorroida & 6 \\
\hline Verbenaceae & Chumbinho & Lantana camara $L$. & Flores & Tosse & 5 \\
\hline Apiaceae & Coentro & Coriandrum sativum $L$. & $\begin{array}{l}\text { Folhas e } \\
\text { sementes }\end{array}$ & Derrame & 3 \\
\hline Brassicaceae & Couve & Brassica oleraceae L. & Folhas & Gastrite & 3 \\
\hline Verbenaceae & $\begin{array}{l}\text { Erva } \\
\text { cidreira }\end{array}$ & Aloysia triphylla Royle & Folhas & Calmante e gripe & 7 \\
\hline Rubiaceae & Jenipapo & Genipa americana $L$. & $\begin{array}{l}\text { Casca dos } \\
\text { frutos }\end{array}$ & Pancada & 2 \\
\hline Annonaceae & Graviola & Annona muricata $L$. & $\begin{array}{l}\text { Sumo das } \\
\text { folhas }\end{array}$ & Câncer & 3 \\
\hline Myrtaceae & Goiaba & Psidium guajava $L$. & Folhas & Diarreia & 4 \\
\hline Lamiaceae & $\begin{array}{c}\text { Hortelã da } \\
\text { folha grossa }\end{array}$ & $\begin{array}{l}\text { Plectranthus } \\
\text { amboinicus (Lour.) } \\
\text { Spreng. }\end{array}$ & Folhas & $\begin{array}{l}\text { Catarro, dor de } \\
\text { ouvido, febre e } \\
\text { bronquite. }\end{array}$ & 7 \\
\hline
\end{tabular}




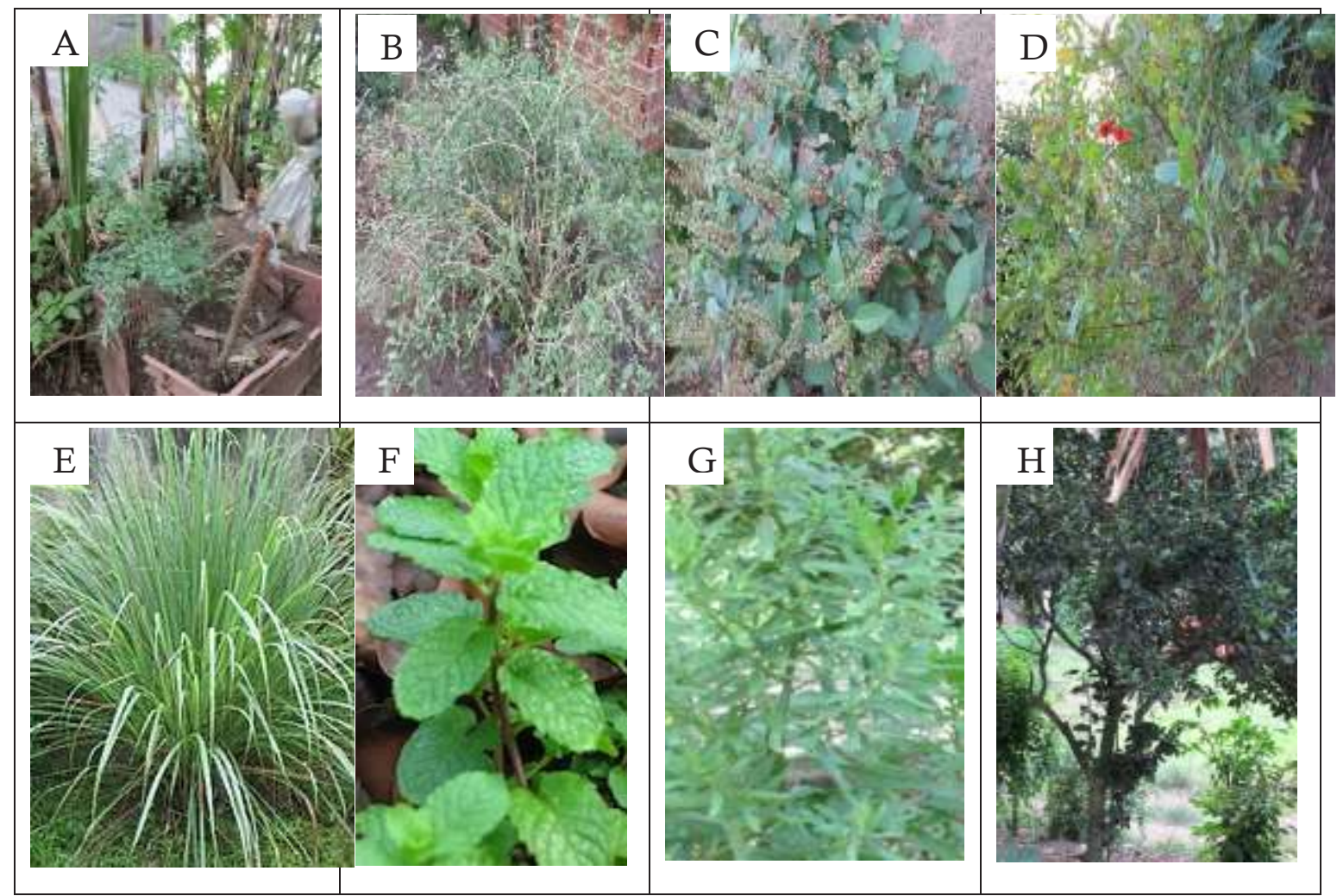

Figura 3: Plantas medicinais mais citadas pelos agricultores (A) arruda, (B) erva cidreira, (C) louro, (D) romã, (E) capim santo, (F) hortelã miúdo, $(\mathrm{G})$ mentruz e $(\mathrm{H})$ laranjeira.

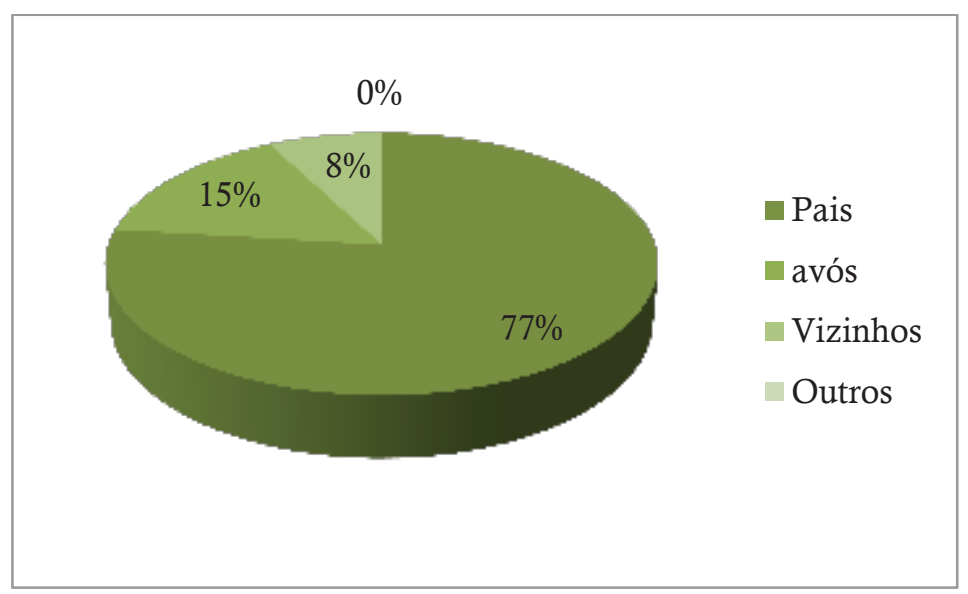

Figura 4. Origem do conhecimento sobre as plantas medicinais dos agricultores da comunidade Moura, Bananeiras, 2013.

moradores sem deixar necessitar de serem utilizados remédios farmacêuticos, contudo a finalidade de cultivar as plantas medicinais são para a contribuição da saúde de famílias da comunidade de forma econômica e natural.

\section{REFERÊNCIAS}

BRITO, M. R.; SENNA-VALLE, L. Plantas medicinais utilizadas na comunidade caiçara da Praia do Sono, Paraty, Rio de Janeiro, Brasil. Acta Botanica Brasilica, v. 25, n. 2, p. 363-372. 2011. CALVACANTE, A. C. P.; SILVA, M. J. R.; SILVA, A. G.; PAULA, A. C.; DINIZ NETO, M. 
A. Levantamento das principais plantas medicinais Comercializadas nas feiras livres dos municípios de Riachão-PB, Campo de Santana-PB e Passa e Fica-RN. In: VI Encontro Nordestino de Etnobiologia e Etnoecologia, Areia-PB, p. 1-6, 2011.

CARAVACA, H. Plantas que curam. Editora Virtual Books Online M\&M Editores Ltda, 2000.

CHAVES, M. S.; DANTAS, F. M.; FONTES, L. S.; CHAVES, R. S.; KINUPP, V. F. Etnobotânica em uma comunidade ribeirinha do Careiro Castanho, AM, Brasil. Cadernos de Agroecologia, v. 6, n. 2, p. 1-6, 2011.

CUNHA, S. A.; BORTOLOTTO, I. M. Etnobotânica de Plantas Medicinais no Assentamento Monjolinho, município de Anastácio, Mato Grosso do Sul, Brasil. Acta Botanica Brasilica, v. 25, n. 3 , p. 685-698, 2011.

GUARIM NETO, G. O saber tradicional pantaneiro: as plantas medicinais e a educação ambiental. Revista eletrônica do Mestrado em Educação Ambiental, v. 17, n. 1, p. 71-89, 2006.

JESUS, N. Z. T.; LIMA, J. C. S.; SILVA, R. M.; ESPINOSA, M. M.; MARTINS, D. T. de O. Levantamento etnobotânico de plantas popularmente utilizadas como antiúlceras e anti-inflamatórias pela comunidade de Pirizal, Nossa Senhora do Livramento-MT, Brasil. Revista Brasileira de Farmacognosia, v. 19, n. 1, p. 130-139, 2009.

LORENZI, H.; MATOS, M. F. J. de A. Plantas medicinais no Brasil - nativas e exóticas. São Paulo: Instituto Plantarum, 2002.

OLIVEIRA, C. J.; ARAÚJO, T. L. Plantas medicinais: usos e crenças de idosos portadores de hipertensão arterial. Revista Eletrônica de Enfermagem, v. 09, n. 01, 2007.

PILLA, M. A. C.; AMOROZO, M. C. de M.; FURLAN, A. Obtenção e uso das plantas medicinais no distrito de Martim Francisco, município de Mogi-Mirim, SP, Brasil. Acta Botanica Brasilica, v. 20, n.4, p. 789-802, 2006.

Phillips, O.; Gentry, A. H.. Th e Useful Plants of Tamboapata, Peru: II. Additional Hypothesis Testing in Quantitative Ethnobotany. Economic Botany 47: p.33-43, 1993.

SANTOS, S. L. D. X.; ALVES, R. R. N.; SANTOS, S. L. D. X.; BARBOSA, J. A. A.; BRA-
SILEIRO, T. F. Plantas utilizadas como medicinais em uma comunidade rural do semi-árido da Paraíba, Nordeste do Brasil. Revista Brasileira de Farmácia, v. 93, n. 1, p. 68-79, 2012. 